\title{
Development and Teaching Application of Virtual Simulation Training System Based on Unity3d
}

\author{
Wei Shen ${ }^{1, *}$ \\ ${ }^{1}$ School of Management and Engineering, Capital University of Economics and Business, Beijing, China \\ ${ }^{*}$ Corresponding author. Email: shw@cueb.edu.cn
}

\begin{abstract}
The project explores a feasible mode of virtual simulation experimental teaching, which can effectively motivate students' interest in joining course learning and improve the quality and effects of teaching. Aiming at defects existing in traditional teaching process, a computer virtual assembly-disassembly teaching system featuring functions such as 3D display, real-time interactions and online evaluation is developed through comprehensive use of software such as 3ds Max, Unity3D and Mysql, so as to effectively remedy deficiencies in traditional practice courses.
\end{abstract}

Keywords: Virtual assembly-disassembly, Unity3D, Simulation teaching.

\section{INTRODUCTION}

Virtual assembly-disassembly simulation teaching refers to the simulation of constitution, working principles and assembly and disassembly of complicated mechanical equipment such as computer with the Virtual Reality(VR) technology. It can also enable a user to make interaction operations with devices such as mouse and data glove. Through comprehensive investigation and manipulation of virtual objects and learning and training of assembly-disassembly maintenance in a simulation environment, effective results of teaching and experiments can be obtained.

\section{DEMAND ANALYSIS AND FUNCTION DESIGN OF THE SYSTEM}

The project makes research based on computer assembly-disassembly experimental teaching in the computer major course Principles of Computer Composition. Due to limits in site, teaching conditions and cost, the teaching instruction is a prevailing teaching mode at present. Specifically, with auxiliary illustration of textbooks, teaching materials and videos, students are led to a lab to watch demonstration of computer assembly and disassembly. In practice, a group comprises 7-8 members and has one computer for exercise. In such mode of teaching and experiment, students are remote from teachers, so they can hardly see or clearly watch teachers' demonstration. In the grouped exercise, one computer is shared by multiple members, so each member only has very limited time to make exercise. In addition, computers used for exercise are mainly eliminated old products of labs and are different from mainstream computer structures at present. Hence, the learned knowledge of assembly and disassembly cannot be accurately matched with practical application.

The above problems can be solved effectively by the teaching mode of virtual simulation experiment. Three functional modules are designed for the system: VR Demonstration, Independent Exercise and Virtual Examination.

\subsection{VR Demonstration Module}

Rather than be retrained by on-site observation and video recording angles, students can independent roaming in three-dimensional virtual scene and observe each part of a computer from 360-degree views. In addition, the system will give corresponding functional illustrations in the form of texts or audios. In teaching demonstration, assembly and disassembly of a computer can be displayed mainly through 3D animation. Students can select observation steps according to demands and learn about it repeatedly.

\subsection{Independent Exercise Module}

Besides traditional devices such as mouse, keyboard and display, students can exercise assembly and disassembly of a computer through external connection of dedicated devices such as data glove and VR glasses. 
In this module, students can make repeated exercise with learned knowledge of assembly and disassembly in a simulated experimental environment, so as to consolidate and deepen their memory. In addition, they can explore other installation sequences at will. After correct installation of hardware, the system will play an audio hint as a judgment standard.

\subsection{Virtual Examination Module}

The system provides assessment of assembly and disassembly operations, so as to realize evaluation and statistical analysis of learning situations and effects. In this module, students will select correct items from multiple components including disturbance items for installation. In the system, scores are preset for correct hardware and installation processes, so scores can be marked according to operation steps and completion situations. Testing results are recorded in corresponding databases.

\section{DEVELOPMENT PROCESSES OF VIRTUAL ASSEMBLY-DISASSEMBLY TEACHING SYSTEM}

Development processes and main work of this project are as follows:

\subsection{D Modeling and Rendering}

At first, a 3D model is established with 3ds Max software. Materials and mappings of different components are given. A scene cartoon is designed and made for teaching demonstration of assembly and disassembly. Vivid texture and light and shadow effects can be added by a V-Ray rendering engine to the 3D model. Hence, defects of 3ds Max default materials in the simulation degree can be effectively remedied. During this course, a hardware model shall be prerendered in 3ds Max, so vivid rendering effects of VRay can be retained.

\subsection{Interaction Design}

An interaction function of the system is completed in Unity3D software. After model introduction and reassignment of mapping, the functions of animation demonstration module, assembly-disassembly exercise module and self-examination module can be realized through coding of C\# scripts. In addition, a user interface for student register and login, and a MySQL database for recording of student performance which is connected by PHP are also added in the system. In the end, the program is published after being tested and optimized.

\subsection{Packaging and Release of the System}

One of the key features of Unity3D is that it can release a product on different platforms. The main mode of releasing the system is network platform, so students can realize independent study and assemblydisassembly exercise on the Internet at any time without being restrained by factors such as equipment and sites. The system is released as a WebGL data packet. An embedded code is generated by Unity3D and then embedded into the website of NetBeans platform, so the Unity3D project can realize normal running in a webpage. In addition, rendering, release and running of Web interactive 3D scenes are realized with the HTML5 marking language. Plus, the system also provides a virtual assembly-disassembly running platform of local immersion type. Specifically, through use of dedicated VR equipment such as data glove, the training can be completed in a mode further similar to natural interactions of humans.

\section{KEY TECHNOLOGIES}

\subsection{Selection of Modeling Accuracy}

Theoretically, the higher the accuracy of a 3D model is, the more real display effects can be reached, and the stronger the immersion sense obtained by a user will be. However, high accuracy will bring great processing burdens to the system, resulting in phenomena such as frame delay and jamming in virtual interaction operations. If a $3 \mathrm{D}$ model is too rough, the sense of immersion brought to the user will be reduced till get vanished. Hence, the significance of application of VR technologies in the teaching system cannot be embodied. Therefore, under the principle of resource optimization, different fineness modeling methods are adopted according to different task demands.

- Fine models are made for key components in need of key illustration, but they are properly simplified. As for parts requiring construction of many polygons in some models such as a concave-convex circuit of a mainboard baseplate and touch points on the CPU bottom, they are directly replaced by high resolution mappings based on in-kind photos.

- Simple models are made for scenes and auxiliary components, so maximum computing resource can be centralized by the system on graphic computation of hardware models during motion and rotation. In this way, problems such as loading delay under frame jamming, shaking and scene switching can be prevented. Hence, fluency in human-computer interactions such as virtual exercise can be enhanced. 


\subsection{Mapping and Exporting of Materials}

Users of a virtual assembly-disassembly teaching system mainly interact with hardware models. Hence, during manufacture, each material in a hardware model is edited separately with the high-quality rendering engine V-Ray; lighting effect changes such as diffuse reflection and attenuation are set; exquisite textures and high-resolution mapping with the resolution ratio $1024 * 1024$ are given; and vividness degree of the model is ensured. The V-Ray material cannot be read by Unity3D, so the hardware model shall be re-rendered in 3ds Max in order to retain V-Ray rendering effects.

\subsection{Implementation of Interaction Function of Information Prompt}

The function is mainly realized in teaching display. When a relevant key component is slid by a mouse, prompts such as name of the component are provided in real time at the lower right corner of the mouse pointer. In case of prompts, users can click the left key of the mouse to check detailed information of the component and can cancel the message box by re-clicking the left key of the mouse.

For implementation of this function, a Collider module shall be added at a corresponding position of the model at first. Then, scripts for popping-up of the message box are edited to designate functions to be implemented when the mouse slides over, leaves and clicks the collider. In this way, information prompt scripts of different components can be loaded on the corresponding models with Collider modules.

\subsection{Design of Database}

The database management tool selected by the virtual teaching assembly-disassembly system is phpMyAdmin. As a graphic management tool of MySQL databases, phpMyAdmin is installed in the Web server and based on PHP, and enables an administrator to manage and operate the MySQL database with a Web interface. Without installation of extra software, teachers can log in a teacher permission account of phpMyAdmin by the browser, realize remote management of the MySQL database, check the quantity, student No., names and exam records of registered students or alter and delete some student information manually. Operations are very convenient.

- Design of this system database is divided into three parts: student information, assembly information and model library.

- A little of student information data is stored. Only identity authentication information such as student No., names and password as well as exam records of students need to be stored.
Data structure is simple and can be established by sheet establishment.

- As for assembly information, parameters such as position relations and assembly-disassembly sequences of each component need to be recorded. In addition, loading of 3D model documents, correct assembly relations and predefined data format files shall be recorded. Through combination of data in the database and a dedicated assembly detection algorithm, whether an operation is correct can be judged. If the installation position and sequence are matched, components can be aligned automatically.

An assembly-disassembly teaching system has high expansibility. The computer and each component are stored in a dedicated 3D model library. New models can be periodically made and input according to current mainstream models. In this way, the problems that inkind teaching tools are outdated and eliminated due to hardware development, upgrading is difficult and cost in maintenance and upgrade is expensive can be solved.

\section{DATA ANALYSIS OF TEACHING EFFECTS}

The course was opened under the epidemic background, so students could not make real computer practice and exercise in school labs. However, a very good opportunity is provided for implementation of the research project in application. For better acquaintance of use effects of the virtual assembly-disassembly teaching system, data analysis was conducted on teaching quality based on multiple dimensions such as learning duration, test scores and questionnaire survey based on a statistics function provided by the online teaching platform. In addition, teaching effects were compared with those obtained through previous virtual training with data gloves in the professional lab.

\subsection{Data Analysis of VR Teaching Demonstration Module}

In this semester, 67 students joined the virtual computer assembly-disassembly training. As shown in final data analysis results, the average rumination ratio for video watching of all the students is $245.73 \%$, and the maximum rumination ratio is $389.41 \%$. Testing performance tends to show linear monotone increasing with the increase of total learning time of the demonstration module. The linear fitting degree of scatter diagrams of both is high $(\mathrm{R} 2=0.8814)$, as shown in Fig.1. 


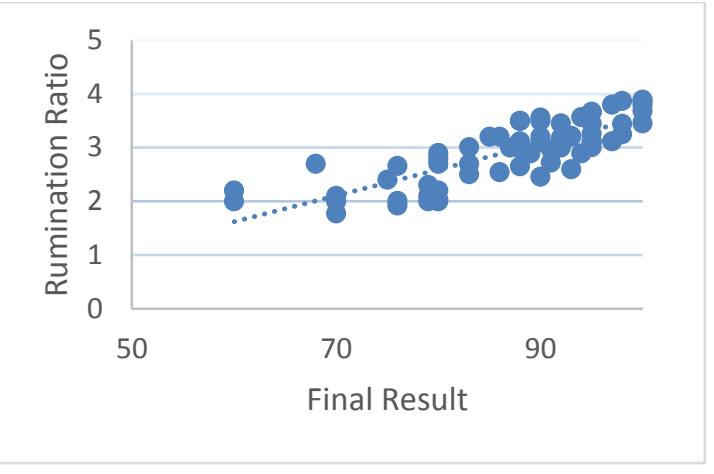

Figure 1 The influence of rumination ratio on final results

\subsection{Data Analysis of Independent Exercise Module}

Students can independently decide times of independent exercise and exercise proposals with different assembly-disassembly sequences. According to statistics results, all the students conducted over 3 exercises, of which $76.12 \%$ of students made 4 exercises, with the higher proportion in the total number. Students with 5 exercises accounted for $14.93 \%$, ranking the second place, as shown in Fig.2. Test scores of students with the exercise times ranking Top 3 were 100, 100 and 99, which are relatively high. Obviously, independent exercise can effectively improve teaching quality and learning performance.

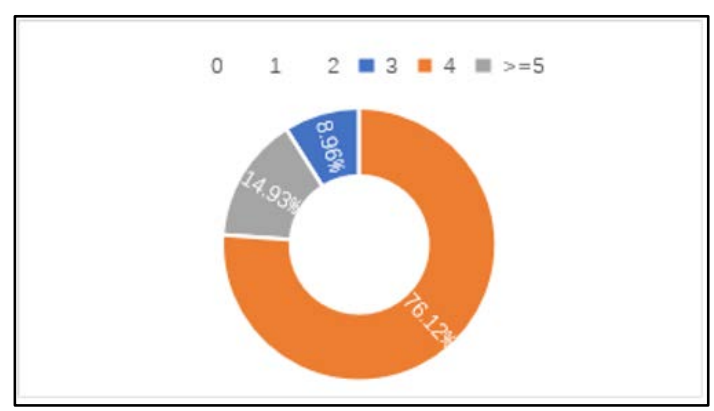

Figure 2 Times of Independent Exercises

\subsection{Comparison of Different Practice Modes}

In the questionnaire survey, results of satisfaction with virtual assembly-disassembly teaching are as follows: $75.3 \%$ of students liked the teaching mode, while $24.7 \%$ of students preferred operations in a real experimental environment mainly because face-to-face communication can better facilitate problem solving as they believed. To a certain extent, this result indicates the indispensable importance of classroom practical teaching.

In the questionnaire survey, 10 students of previous grades, who had conducted virtual training with data gloves in professional labs, were asked to join the user experience comparison. As felt by them in general, it is interesting to use professional VR devices such as data glove, and the sense of immersion is stronger in operations; there is no significant difference between the this mode and the online exercise with use of mouse and keyboard in accuracy of task completion and recognition rates of errors; however, interaction complexity with use of data gloves is slightly higher, and there are also limits in quantity, cost, use sites, etc. In comprehensive view of above factors, as believed by the students, the current set of webpage embedded assembly-disassembly training system has higher practicability and higher values in teaching promotion.

\section{CONCLUSION}

$\mathrm{VR}$ is an emerging type of science. Its extensive application in education is limited by many factors at present. However, because of its "Immersion", "interaction" and "imagination" characteristics, it can help teaching staffs formulate a brand-new and varying teaching environment and learning method, so as to provide learners with an interactive and directive learning environment and method based on independent exploration. In this way, students will be further motivated to join course learning, and quality and effects can be improved in different aspects of teaching and learning. In order to create more natural and immersive 3D interaction experience, the system will try natural interactions in subsequent upgrading and improvement as planned. Specifically, double-handed exercise without suspended operations, especially motions such as crossing and position changing of both hands, can be realized through gesture recognition technologies in combination with algorithms such as deep learning. Through improvement based on algorithms, user experience can be greatly improved, and feasible reference can be provided for teaching reform of more courses.

\section{ACKNOWLEDGMENTS}

This research was financially supported by National Social Science Fund of China in 2019 (No.19BXW120, Title: The Study of Data Driven Smart Media's Influence on Adolescent's Behavior Patterns).

\section{REFERENCES}

[1] Yuling Yan. Design and implementation of virtual maintenance teaching system based on unity3d[J]. Design and Application Research. 2019(11):128130

[2] Siqun Ma. Research on virtual dismounting training system of EMU bogies based on unity3d[J]. Computer application. 2017(4):33-37

[3] Zhiwen Yang. Design and implementation of virtual assembly and disassembly teaching system of 
modular fixture based on unity3d[J]. Journal of Hebei University of Technology. 2018(4):8-16

[4] Xiaolin Yi. Research on Virtual Laboratory of computer system on the Internet[J]. Computer Engineering. 2002(11): 243-258
[5] Jayaram S.et al. VADE: A virtual assembly design environment[J]. America: IEEE Computer Graphics and Application, 1999: 43-45

[6] Wenjin Tao. Augmented, Virtual, and Mixed Reality Applications in Advanced Manufacturing[J]. America: Missouri University of Science and Technology, 2019: 